\title{
ASYMPTOTIC ANALYSIS OF THE RADIAL MINIMIZERS OF AN ENERGY FUNCTIONAL *
}

\author{
YUTIAN LEI ${ }^{\dagger}$
}

Abstract. The author proves the $W^{1, p}$ convergence of the radial minimizers $u_{\varepsilon}=\left(u_{\varepsilon 1}, u_{\varepsilon 2}, u_{\varepsilon 3}\right)$ of an energy functional as $\varepsilon \rightarrow 0$, and the zeros of $u_{\varepsilon 1}^{2}+u_{\varepsilon 2}^{2}$ are located roughly. In addition, the estimates of the convergent rate of $u_{\varepsilon 3}^{2}$ are presented.

1. Introduction. Let $B=\left\{x \in R^{2} ; x_{1}^{2}+x_{2}^{2}<1\right\}$. Denote $S^{1}=\left\{x \in R^{3} ; x_{1}^{2}+\right.$ $\left.x_{2}^{2}=1, x_{3}=0\right\}$ and $S^{2}=\left\{x \in R^{3} ; x_{1}^{2}+x_{2}^{2}+x_{3}^{2}=1\right\}$. Let $g(x)=\left(e^{i d \theta}, 0\right)$ where $x=(\cos \theta, \sin \theta)$ on $\partial B, d \in N$. We concern with the minimizer of the energy functional

$$
E_{\varepsilon}(u, B)=\frac{1}{p} \int_{B}|\nabla u|^{p} d x+\frac{1}{2 \varepsilon^{p}} \int_{B} u_{3}^{2} d x \quad(p>2)
$$

in the function class

$$
W=\left\{u(x)=\left(\sin f(r) e^{i d \theta}, \cos f(r)\right) \in W^{1, p}\left(B, S^{2}\right) ;\left.u\right|_{\partial B}=g\right\},
$$

which is named the radial minimizer of $E_{\varepsilon}(u, B)$.

When $p=2$, the functional $E_{\varepsilon}(u, B)$ was introduced in the study of some simplified model of high-energy physics, which controls the statics of planar ferromagnets and antiferromagnets (see [3][4]). The asymptotic behavior of minimizers of $E_{\varepsilon}(u, B)$ has been considered by Fengbo Hang and Fanghua Lin in [2]. (In particular, they discussed the asymptotic behavior of the radial minimizer of $E_{\varepsilon}(u, B)$ in $\S 5$.)

In this paper, we always assume $p>2$. As in [2][3] and [4], we are interested in the behavior of minimizers of $E_{\varepsilon}(u, B)$ as $\varepsilon \rightarrow 0$. We will prove the $W_{l o c}^{1, p}$ convergence of the radial minimizers. In addition, some estimates of the convergent rate of the radial minimizer will be presented and we will discuss the location of the points where $u_{3}^{2}=1$.

In polar coordinates, for $u(x)=\left(\sin f(r) e^{i d \theta}, \cos f(r)\right)$, we have

$$
\begin{gathered}
|\nabla u|=\left(f_{r}^{2}+d^{2} r^{-2} \sin ^{2} f\right)^{1 / 2} \\
\int_{B}|\nabla u|^{p} d x=2 \pi \int_{0}^{1} r\left(f_{r}^{2}+d^{2} r^{-2} \sin ^{2} f\right)^{p / 2} d r .
\end{gathered}
$$

If we denote

$$
V=\left\{f \in W_{l o c}^{1, p}(0,1] ; r^{1 / p} f_{r}, r^{(1-p) / p} \sin f \in L^{p}(0,1), f(r) \geq 0, f(1)=\frac{\pi}{2}\right\},
$$

then $V=\left\{f(r) ; u(x)=\left(\sin f(r) e^{i d \theta}, \cos f(r)\right) \in W\right\}$.

\footnotetext{
*Received September 16, 2002; accepted for publication June 19, 2003.

${ }^{\dagger}$ Department of Mathematics, Nanjing Normal University, Nanjing, Jiangsu, 210097, P.R.China (lythxl@163.com).
} 
REMARK. $V \subset\{f \in C[0,1] ; f(0)=0\}$.

In fact, if $f \in V$, denote $h(r)=f\left(r^{1+\frac{1}{p-2}}\right)$, then

$$
\begin{aligned}
\int_{0}^{1}\left|h^{\prime}(r)\right|^{p} d r & =\left(1+\frac{1}{p-2}\right)^{p} \int_{0}^{1}\left|f^{\prime}\left(r^{1+\frac{1}{p-2}}\right)\right|^{p} r^{\frac{p}{p-2}} d r \\
& =\left(1+\frac{1}{p-2}\right)^{p}\left(1-\frac{1}{p-1}\right) \int_{0}^{1} s\left|f^{\prime}(s)\right|^{p} d s<\infty
\end{aligned}
$$

which implies that $h(r) \in C[0,1]$ and hence $f(r) \in C[0,1]$.

Suppose $f(0)>0$, then $f(r) \geq s>0$ for $r \in[0, t)$ with $t>0$ sufficiently small. This implies that $\sin f(r) \geq \sin s>0$. Noting $p>2$, we have

$$
\int_{0}^{1} r^{1-p} \sin ^{p} f d r \geq \sin ^{p} s \int_{0}^{t} r^{1-p} d r=\infty
$$

which contradicts $r^{1 / p-1} \sin f \in L^{p}(0,1)$. Therefore $f(0)=0$.

Substituting $u(x)=\left(\sin f(r) e^{i d \theta}, \cos f(r)\right) \in W$ into $E_{\varepsilon}(u, B)$ we obtain

$$
E_{\varepsilon}(u, B)=2 \pi E_{\varepsilon}(f,(0,1)),
$$

where

$$
E_{\varepsilon}(f,(0,1))=\int_{0}^{1}\left[\frac{1}{p}\left(f_{r}^{2}+d^{2} r^{-2} \sin ^{2} f\right)^{p / 2}+\frac{1}{2 \varepsilon^{p}} \cos ^{2} f\right] r d r .
$$

This shows that $u=\left(\sin f(r) e^{i d \theta}, \cos f(r)\right) \in W$ is the minimizer of $E_{\varepsilon}(u, B)$ if and only if $f(r) \in V$ is the minimizer of $E_{\varepsilon}(f,(0,1))$. Applying the direct method in the calculus of variations we can see that the functional $E_{\varepsilon}(u, B)$ achieves its minimum on $W$ by a function $u_{\varepsilon}(x)=\left(\sin f_{\varepsilon}(r) e^{i d \theta}, \cos f_{\varepsilon}(r)\right)$, hence $f_{\varepsilon}(r)$ is the minimizer of $E_{\varepsilon}(f,(0,1))$.

We will prove the following

THeOREM 1.1. Let $u_{\varepsilon}$ be a radial minimizer of $E_{\varepsilon}(u, B)$ on $W$. Then for any $\gamma \in(0,1)$, there exists a constant $h=h(\gamma)$ which is independent of $\varepsilon \in(0,1)$ such that $Z_{\varepsilon}=\left\{x \in B ;\left|u_{\varepsilon 3}\right|>\gamma\right\} \subset B(0, h \varepsilon)$.

This theorem shows that all the points where $u_{\varepsilon 3}^{2}=1$ are contained in $B(0, h \varepsilon)$. Hence as $\varepsilon \rightarrow 0$, these points converge to 0 .

THEOREM 1.2. Let $u_{\varepsilon}(x)=\left(\sin f_{\varepsilon}(r) e^{i d \theta}, \cos f_{\varepsilon}(r)\right)$ be a radial minimizer of $E_{\varepsilon}(u, B)$ on $W$. Then

$$
\lim _{\varepsilon \rightarrow 0} u_{\varepsilon}=\left(e^{i d \theta}, 0\right), \quad \text { in } \quad W^{1, p}\left(K, R^{3}\right)
$$

for any compact subset $K \subset \bar{B} \backslash\{0\}$.

THEOREM 1.3. (convergent rate) Let $u_{\varepsilon}(x)=\left(\sin f_{\varepsilon}(r) e^{i d \theta}, \cos f_{\varepsilon}(r)\right)$ be a radial minimizer of $E_{\varepsilon}(u, B)$ on $W$. Then for any $\eta \in(0,1)$, there exist $C, \varepsilon_{0}>0$ such that as $\varepsilon \in\left(0, \varepsilon_{0}\right)$,

$$
\int_{\eta}^{1} r\left[\left(f_{\varepsilon}^{\prime}\right)^{p}+\frac{1}{\varepsilon^{p}} \cos ^{2} f_{\varepsilon}\right] d r \leq C \varepsilon^{p} .
$$




$$
\sup _{x \in K}\left|u_{\varepsilon 3}(x)\right| \leq C \varepsilon^{\frac{p-2}{2}} .
$$

Here $K=\bar{B} \backslash B(0, \eta)$.

The proof of Theorem 1.1 will be given in $\S 2$. In $\S 3$,we will set up the uniform estimate of $E_{\varepsilon}\left(u_{\varepsilon}, K\right)$ which implies the conclusion of Theorem 1.2 (see $\S 4$ ). By virtue of the uniform estimate we can also derive the proof of Theorem 1.3 in $\S 5$.

\section{Proof of Theorem 1.1.}

Proposition 2.1. Let $f_{\varepsilon}$ be a minimizer of $E_{\varepsilon}(f,(0,1))$. Then

$$
E_{\varepsilon}\left(f_{\varepsilon},(0,1)\right) \leq C \varepsilon^{2-p}
$$

with a constant $C$ independent of $\varepsilon \in(0,1)$.

Proof. Denote

$$
I(\varepsilon, R)=\operatorname{Min}\left\{\int_{0}^{R}\left[\frac{1}{p}\left(f_{r}^{2}+\frac{d^{2}}{r^{2}} \sin ^{2} f\right)^{\frac{p}{2}}+\frac{1}{2 \varepsilon^{p}} \cos ^{2} f\right] r d r ; f \in V_{R}\right\}
$$

where

$$
\begin{aligned}
V_{R}=\{f(r) \quad & \in W_{l o c}^{1, p}(0, R] ; f(R)=\frac{\pi}{2}, \\
& \left.\sin f(r) r^{\frac{1}{p}-1}, f^{\prime}(r) r^{\frac{1}{p}} \in L^{p}(0, R)\right\} .
\end{aligned}
$$

Then

$$
\begin{aligned}
& I(\varepsilon, 1)=E_{\varepsilon}\left(f_{\varepsilon},(0,1)\right) \\
= & \frac{1}{p} \int_{0}^{1} r\left(\left(f_{\varepsilon}\right)_{r}^{2}+d^{2} r^{-2}\left(\sin f_{\varepsilon}\right)^{2}\right)^{p / 2} d r+\frac{1}{2 \varepsilon^{p}} \int_{0}^{1} r \cos ^{2} f_{\varepsilon} d r \\
= & \frac{1}{p} \int_{0}^{1 / \varepsilon} \varepsilon^{2-p} s\left(\left(f_{\varepsilon}\right)_{s}^{2}+d^{2} s^{-2} \sin ^{2} f_{\varepsilon}\right)^{p / 2} d s+\frac{1}{2 \varepsilon^{p}} \int_{0}^{\varepsilon^{-1}} \varepsilon^{2} s \cos ^{2} f_{\varepsilon} d s \\
= & \varepsilon^{2-p} I\left(1, \varepsilon^{-1}\right) .
\end{aligned}
$$

Let $f_{1}$ be the minimizer for $I(1,1)$ and define

$$
f_{2}=f_{1}, \quad \text { as } \quad 0<s<1 ; \quad f_{2}=\frac{\pi}{2}, \quad \text { as } \quad 1 \leq s \leq \varepsilon^{-1}
$$

We have

$$
\begin{aligned}
I\left(1, \varepsilon^{-1}\right) \leq & \frac{1}{p} \int_{0}^{\varepsilon^{-1}} s\left[\left(f_{2}^{\prime}\right)^{2}+d^{2} s^{-2} \sin ^{2} f_{2}\right]^{p / 2} d s+\frac{1}{2} \int_{0}^{\varepsilon^{-1}} s \cos ^{2} f_{2} d s \\
\leq & \frac{1}{p} \int_{1}^{\varepsilon^{-1}} s^{1-p} d^{p} d s+\frac{1}{p} \int_{0}^{1} s\left(\left(f_{1}^{\prime}\right)^{2}+d^{2} s^{-2} \sin ^{2} f_{1}\right)^{p / 2} d s \\
& +\frac{1}{2} \int_{0}^{1} s \cos ^{2} f_{1} d s \\
= & \frac{d^{p}}{p(p-2)}\left(1-\varepsilon^{p-2}\right)+I(1,1) \leq \frac{d^{p}}{p(p-2)}+I(1,1)=C .
\end{aligned}
$$


Substituting into (2.1) follows the conclusion of Proposition 2.1.

By the embedding theorem we first derive from $\left|u_{\varepsilon}\right|=1$ and proposition 2.1 the following

Proposition 2.2. Let $u_{\varepsilon}$ be a radial minimizer of $E_{\varepsilon}(u, B)$. Then there exists a constant $C$ independent of $\varepsilon \in(0,1)$ such that

$$
\left|u_{\varepsilon}(x)-u_{\varepsilon}\left(x_{0}\right)\right| \leq C \varepsilon^{(2-p) / p}\left|x-x_{0}\right|^{1-2 / p}, \quad \forall x, x_{0} \in B .
$$

As a corollary of Proposition 2.1 we have

Proposition 2.3. Let $u_{\varepsilon}$ be a radial minimizer of $E_{\varepsilon}(u, B)$. Then for some constant $C$ independent of $\varepsilon \in(0,1]$ such that

$$
\frac{1}{\varepsilon^{2}} \int_{B} u_{\varepsilon 3}^{2} d x \leq C .
$$

Based on Proposition 2.2, we have the interesting result:

Proposition 2.4. Let $u_{\varepsilon}$ be a radial minimizer of $E_{\varepsilon}(u, B)$. Then for any $\gamma \in(0,1)$, there exist positive constants $\lambda, \mu$ independent of $\varepsilon \in(0,1)$ such that if

$$
\frac{1}{\varepsilon^{2}} \int_{B \cap B^{2 l \varepsilon}} u_{\varepsilon 3}^{2} d x \leq \mu
$$

where $B^{2 l \varepsilon}$ is some disc of radius $2 l \varepsilon$ with $l \geq \lambda$, then

$$
\left|u_{\varepsilon 3}(x)\right| \leq \gamma, \quad \forall x \in B \cap B^{l \varepsilon}
$$

Proof. First we observe that there exists a constant $\beta>0$ such that for any $x \in B$ and $0<\rho \leq 1$,

$$
\operatorname{mes}(B \cap B(x, \rho)) \geq \beta \rho^{2} .
$$

To prove the proposition, we choose

$$
\lambda=\left(\frac{\gamma}{2 C}\right)^{\frac{p}{p-2}}, \mu=\frac{\beta}{4}\left(\frac{1}{2 C}\right)^{\frac{2 p}{p-2}} \gamma^{2+\frac{2 p}{p-2}}
$$

where $\mathrm{C}$ is the constant in Proposition 2.2.

Suppose that there is a point $x_{0} \in B \cap B^{l \varepsilon}$ such that (2.3) is not true, i.e.

$$
\left|u_{\varepsilon 3}\left(x_{0}\right)\right|>\gamma .
$$

Then applying Proposition 2.2 we have

$$
\begin{aligned}
\left|u_{\varepsilon}(x)-u_{\varepsilon}\left(x_{0}\right)\right| & \leq C \varepsilon^{(2-p) / p}\left|x-x_{0}\right|^{1-2 / p} \leq C \varepsilon^{(2-p) / p}(\lambda \varepsilon)^{1-2 / p} \\
& =C \lambda^{1-2 / p}=\frac{\gamma}{2}, \quad \forall x \in B\left(x_{0}, \lambda \varepsilon\right)
\end{aligned}
$$

which implies that

$$
\left|u_{\varepsilon 3}(x)-u_{\varepsilon 3}\left(x_{0}\right)\right| \leq \frac{\gamma}{2} .
$$


Noticing (2.4), we obtain

$$
\left|u_{\varepsilon 3}(x)\right|^{2} \geq\left[\left|u_{\varepsilon 3}\left(x_{0}\right)\right|-\frac{\gamma}{2}\right]^{2}>\frac{\gamma^{2}}{4}, \quad \forall x \in B\left(x_{0}, \lambda \varepsilon\right) .
$$

Hence

$$
\begin{gathered}
\int_{B\left(x_{0}, \lambda \varepsilon\right) \cap B} u_{\varepsilon 3}^{2} d x>\frac{\gamma^{2}}{4} \operatorname{mes}\left(B \cap B\left(x_{0}, \lambda \varepsilon\right)\right) \\
\geq \beta \frac{\gamma^{2}}{4}(\lambda \varepsilon)^{2}=\beta \frac{\gamma^{2}}{4}\left(\frac{\gamma}{2 C}\right)^{\frac{2 p}{p-2}} \varepsilon^{2}=\mu \varepsilon^{2} .
\end{gathered}
$$

Since $x_{0} \in B^{l \varepsilon} \cap B$, and $\left(B\left(x_{0}, \lambda \varepsilon\right) \cap B\right) \subset\left(B^{2 l \varepsilon} \cap B\right),(2.5)$ implies

$$
\int_{B^{2 l \varepsilon} \cap B} u_{\varepsilon 3}^{2} d x>\mu \varepsilon^{2},
$$

which contradicts $(2.2)$ and thus the proposition is proved.

To find the points where $u_{\varepsilon 3}^{2}=1$ based on Proposition 2.4, we may take (2.2) as the ruler to distinguish the discs of radius $\lambda \varepsilon$ which contain these points.

Let $u_{\varepsilon}$ be a radial minimizer of $E_{\varepsilon}(u, B)$. Given $\gamma \in(0,1)$. Let $\lambda, \mu$ be constants in Proposition 2.4 corresponding to $\gamma$. If

$$
\frac{1}{\varepsilon^{2}} \int_{B\left(x^{\varepsilon}, 2 \lambda \varepsilon\right) \cap B} u_{\varepsilon 3}^{2} d x \leq \mu,
$$

then $B\left(x^{\varepsilon}, \lambda \varepsilon\right)$ is called $\gamma-$ good disc, or simply good disc. Otherwise $B\left(x^{\varepsilon}, \lambda \varepsilon\right)$ is called $\gamma-$ bad disc or simply bad disc.

Now suppose that $\left\{B\left(x_{i}^{\varepsilon}, \lambda \varepsilon\right), i \in I\right\}$ is a family of discs satisfying

$$
\begin{gathered}
(i): x_{i}^{\varepsilon} \in B, i \in I ; \quad(i i): B \subset \cup_{i \in I} B\left(x_{i}^{\varepsilon}, \lambda \varepsilon\right) ; \\
(i i i): B\left(x_{i}^{\varepsilon}, \lambda \varepsilon / 4\right) \cap B\left(x_{j}^{\varepsilon}, \lambda \varepsilon / 4\right)=\emptyset, i \neq j .
\end{gathered}
$$

Denote

$$
J_{\varepsilon}=\left\{i \in I ; B\left(x_{i}^{\varepsilon}, \lambda \varepsilon\right) \text { is a bad disc }\right\}
$$

Proposition 2.5. There exists a positive integer $N$ such that the number of bad discs $\operatorname{Card} J_{\varepsilon} \leq N$.

Proof. Since (2.6) implies that every point in $B$ can be covered by finite, say $m$ (independent of $\varepsilon$ ) discs, from Proposition 2.3 and the definition of bad discs, we have

$$
\begin{aligned}
& \mu \varepsilon^{2} \operatorname{Card}_{\varepsilon} \leq \sum_{i \in J_{\varepsilon}} \int_{B\left(x_{i}^{\varepsilon}, 2 \lambda \varepsilon\right) \cap B} u_{\varepsilon 3}^{2} d x \\
\leq & m \int_{\cup_{i \in J_{\varepsilon}} B\left(x_{i}^{\varepsilon}, 2 \lambda \varepsilon\right) \cap B} u_{\varepsilon 3}^{2} d x \leq m \int_{B} u_{\varepsilon 3}^{2} d x \leq m C \varepsilon^{2}
\end{aligned}
$$

and hence Card $J_{\varepsilon} \leq \frac{m C}{\mu} \leq N$.

Applying TheoremIV.1 in [1], we may modify the family of bad discs such that the new one, denoted by $\left\{B\left(x_{i}^{\varepsilon}, h \varepsilon\right) ; i \in J\right\}$, satisfies

$$
\cup_{i \in J_{\varepsilon}} B\left(x_{i}^{\varepsilon}, \lambda \varepsilon\right) \subset \cup_{i \in J} B\left(x_{i}^{\varepsilon}, h \varepsilon\right), \quad \lambda \leq h ; \quad \text { Card } J \leq \text { Card } J_{\varepsilon},
$$




$$
\left|x_{i}^{\varepsilon}-x_{j}^{\varepsilon}\right|>8 h \varepsilon, i, j \in J, i \neq j .
$$

The last condition implies that every two discs in the new family are not intersected. From Proposition 2.4 it is deduced that all the points where $\left|u_{\varepsilon 3}\right|=1$ are contained in these finite, disintersected bad discs.

Now we prove our main result of this section.

THEOREM 2.6. Let $u_{\varepsilon}$ be a radial minimizer of $E_{\varepsilon}(u, B)$. Then for any $\gamma \in(0,1)$, there exists a constant $h=h(\gamma)$ independent of $\varepsilon \in(0,1)$ such that $Z_{\varepsilon}=\{x \in$ $\left.B ;\left|u_{\varepsilon 3}(x)\right|>\gamma\right\} \subset B(0, h \varepsilon)$.

Proof. Suppose there exists a point $x_{0} \in Z_{\varepsilon}$ such that $x_{0} \bar{\in} B(0, h \varepsilon)$. Then all points on the circle $S_{0}=\left\{x \in B ;|x|=\left|x_{0}\right|\right\}$ satisfy

$$
u_{\varepsilon 3}^{2}(x)=\cos ^{2} f_{\varepsilon}(|x|)=\cos ^{2} f_{\varepsilon}\left(\left|x_{0}\right|\right)=u_{\varepsilon 3}^{2}\left(x_{0}\right)>\gamma^{2} .
$$

By virtue of Proposition 2.4 we can see that all points on $S_{0}$ are contained in bad discs. However, since $\left|x_{0}\right| \geq h \varepsilon, S_{0}$ can not be covered by a single bad disc. As a result, $S_{0}$ has to be covered by at least two bad disintersected discs. This is impossible.

This theorem implies that all the zeros of $u_{\varepsilon 1}^{2}+u_{\varepsilon 2}^{2}$ are contained in $B(0, h \varepsilon)$. In particular the zeros are converge to 0 as $\varepsilon \rightarrow 0$.

3. Uniform estimate. Let $u_{\varepsilon}(x)=\left(\sin f_{\varepsilon}(r) e^{i d \theta}, \cos f_{\varepsilon}(r)\right)$ be a radial minimizer of $E_{\varepsilon}(u, B)$, namely $f_{\varepsilon}$ be a minimizer of

$$
E_{\varepsilon}(f,(0,1))=\frac{1}{p} \int_{0}^{1}\left(f_{r}^{2}+d^{2} r^{-2} \sin ^{2} f\right)^{p / 2} r d r+\frac{1}{2 \varepsilon^{p}} \int_{0}^{1} \cos ^{2} f r d r
$$

in $V$. From Proposition 2.1, we have

$$
E_{\varepsilon}\left(f_{\varepsilon},(0,1)\right) \leq C \varepsilon^{2-p}
$$

for some constant $C$ independent of $\varepsilon \in(0,1)$.

In this section we further prove that for any $\eta \in(0,1)$, there exists a constant $C(\eta)$ such that

$$
E_{\varepsilon}\left(f_{\varepsilon} ; \eta\right):=E_{\varepsilon}\left(f_{\varepsilon},(\eta, 1)\right) \leq C(\eta)
$$

for $\varepsilon \in\left(0, \varepsilon_{0}\right)$ with small $\varepsilon_{0}>0$. Based on the estimate (3.2) and Theorem 2.6, we may obtain better convergence for minimizers, namely the $W_{l o c}^{1, p}$ convergence.

To establish (3.2) we first prove

Proposition 3.1. Given $\eta \in(0,1)$. There exist constants

$$
\eta_{j} \in\left[\frac{(j-1) \eta}{N+1}, \frac{j \eta}{N+1}\right],(N=[p])
$$

and $C_{j}$, such that

$$
E_{\varepsilon}\left(f_{\varepsilon}, \eta_{j}\right) \leq C_{j} \varepsilon^{j-p}
$$

for $j=2, \ldots, N$, where $\varepsilon \in\left(0, \varepsilon_{0}\right)$. 
Proof. For $j=2$, the inequality (3.3) is just the one in Proposition 2.1.

Suppose that (3.3) holds for all $j \leq n$. Then we have, in particular

$$
E_{\varepsilon}\left(f_{\varepsilon} ; \eta_{n}\right) \leq C_{n} \varepsilon^{n-p} .
$$

If $n=N$ then we are done. Suppose $n<N$. We want to prove (3.3) for $j=n+1$.

Obviously (3.4) implies

$$
\begin{aligned}
& \frac{1}{p} \int_{\frac{n \eta}{N+1}}^{\frac{(n+1) \eta}{N+1}}\left[\left(f_{\varepsilon}\right)_{r}^{2}+d^{2} r^{-2} \sin ^{2} f_{\varepsilon}\right]^{p / 2} r d r \\
& +\frac{1}{4 \varepsilon^{p}} \int_{\frac{n \eta}{N+1}}^{\frac{(n+1) \eta}{N+1}} \cos ^{2} f_{\varepsilon} r d r \leq C_{n} \varepsilon^{n-p}
\end{aligned}
$$

from which we see by integral mean value theorem that there exists

$$
\eta_{n+1} \in\left[\frac{n \eta}{N+1}, \frac{(n+1) \eta}{N+1}\right]
$$

such that

$$
\begin{gathered}
{\left[\left(f_{\varepsilon}\right)_{r}^{2}+d^{2} r^{-2} \sin ^{2} f_{\varepsilon}\right]_{r=\eta_{n+1}}^{p / 2} \leq C_{n} \varepsilon^{n-p},} \\
{\left[\frac{1}{\varepsilon^{p}} \cos ^{2} f_{\varepsilon}\right]_{r=\eta_{n+1}} \leq C_{n} \varepsilon^{n-p} .}
\end{gathered}
$$

Consider the functional

$$
E\left(\rho, \eta_{n+1}\right)=\frac{1}{p} \int_{\eta_{n+1}}^{1}\left(\rho_{r}^{2}+1\right)^{p / 2} d r+\frac{1}{\varepsilon^{p}} \int_{\eta_{n+1}}^{1} \cos ^{2} \rho d r .
$$

It is easy to prove that the minimizer $\rho_{1}$ of $E\left(\rho, \eta_{n+1}\right)$ in $W_{f_{\varepsilon}}^{1, p}\left(\left(\eta_{n+1}, 1\right), R^{+}\right)$exists and satisfies

$$
\begin{gathered}
-\varepsilon^{p}\left(v^{(p-2) / 2} \rho_{r}\right)_{r}=\sin 2 \rho, \quad \text { in }\left(\eta_{n+1}, 1\right) \\
\left.\rho\right|_{r=\eta_{n+1}}=f_{\varepsilon},\left.\quad \rho\right|_{r=1}=f_{\varepsilon}(1)=\frac{\pi}{2}
\end{gathered}
$$

where $v=\rho_{r}^{2}+1$. It follows from the maximum principle that $\rho_{1} \leq \pi / 2$ and

$$
\sin ^{2} \rho(r) \geq \sin ^{2} \rho\left(\eta_{n+1}\right)=\sin ^{2} f_{\varepsilon}\left(\eta_{n+1}\right)=1-\cos ^{2} f_{\varepsilon}\left(\eta_{n+1}\right) \geq 1-\gamma^{2}
$$

the last inequality of which is implied by Theorem 2.6.

Applying (3.4) we see easily that

$$
E\left(\rho_{1} ; \eta_{n+1}\right) \leq E\left(f_{\varepsilon} ; \eta_{n+1}\right) \leq C_{n} E_{\varepsilon}\left(f_{\varepsilon} ; \eta_{n+1}\right) \leq C_{n} \varepsilon^{n-p}
$$

for $\varepsilon \in\left(0, \varepsilon_{0}\right)$ with $\varepsilon_{0}>0$ sufficiently small.

Now choosing a smooth function $\zeta(r)$ such that $\zeta=1$ on $(0, \eta), \zeta=0$ near $r=1$, multiplying $(3.7)$ by $\zeta \rho_{r}\left(\rho=\rho_{1}\right)$ and integrating over $\left(\eta_{n+1}, 1\right)$ we obtain

$$
\begin{aligned}
\left.v^{(p-2) / 2} \rho_{r}^{2}\right|_{r=\eta_{n+1}} & +\int_{\eta_{n+1}}^{1} v^{(p-2) / 2} \rho_{r}\left(\zeta_{r} \rho_{r}+\zeta \rho_{r r}\right) d r \\
& =\frac{1}{\varepsilon^{p}} \int_{\eta_{n+1}}^{1} \sin 2 \rho \zeta \rho_{r} d r
\end{aligned}
$$


Using (3.10) we have

$$
\begin{aligned}
& \left|\int_{\eta_{n+1}}^{1} v^{(p-2) / 2} \rho_{r}\left(\zeta_{r} \rho_{r}+\zeta \rho_{r r}\right) d r\right| \\
\leq & \int_{\eta_{n+1}}^{1} v^{(p-2) / 2}\left|\zeta_{r}\right| \rho_{r}^{2} d r+\frac{1}{p}\left|\int_{\eta_{n+1}}^{1}\left(v^{p / 2} \zeta\right)_{r} d r-\int_{\eta_{n+1}}^{1} v^{p / 2} \zeta_{r} d r\right| \\
\leq & C \int_{\eta_{n+1}}^{1} v^{p / 2} d r+\left.\frac{1}{p} v^{p / 2}\right|_{r=\eta_{n+1}}+\frac{C}{p} \int_{\eta_{n+1}}^{1} v^{p / 2} d r \\
\leq & C \int_{\eta_{n+1}}^{1} v^{p / 2} d r+\left.\frac{1}{p} v^{p / 2}\right|_{r=\eta_{n+1}} \leq C_{n} \varepsilon^{n-p}+\left.\frac{1}{p} v^{p / 2}\right|_{r=\eta_{n+1}}
\end{aligned}
$$

and using (3.6)(3.10) we have

$$
\begin{aligned}
& \quad\left|\frac{1}{\varepsilon^{p}} \int_{\eta_{n+1}}^{1} \zeta \rho_{r} \sin 2 \rho d r\right|=\frac{1}{\varepsilon^{p}}\left|\int_{\eta_{n+1}}^{1} \zeta_{r} \cos ^{2} \rho d r-\int_{\eta_{n+1}}^{1}\left(\zeta \cos ^{2} \rho\right)_{r} d r\right| \\
& \leq\left.\frac{1}{\varepsilon^{p}} \cos ^{2} \rho\right|_{r=\eta_{n+1}}+\frac{C}{\varepsilon^{p}} \int_{\eta_{n+1}}^{1} \cos ^{2} \rho d r \leq C_{n} \varepsilon^{n-p} .
\end{aligned}
$$

Combining (3.11) with (3.12)(3.13) yields

$$
\left.v^{(p-2) / 2} \rho_{r}^{2}\right|_{r=\eta_{n+1}} \leq C_{n} \varepsilon^{n-p}+\left.\frac{1}{p} v^{p / 2}\right|_{r=\eta_{n+1}} .
$$

Hence

$$
\begin{aligned}
\left.v^{p / 2}\right|_{r=\eta_{n+1}} & =\left.v^{(p-2) / 2}\left(\rho_{r}^{2}+1\right)\right|_{r=\eta_{n+1}}=\left.v^{(p-2) / 2} \rho_{r}^{2}\right|_{r=\eta_{n+1}}+\left.v^{(p-2) / 2}\right|_{r=\eta_{n+1}} \\
& \leq C_{n} \varepsilon^{n-p}+\left.\frac{1}{p} v^{p / 2}\right|_{r=\eta_{n+1}}+\left.v^{(p-2) / 2}\right|_{r=\eta_{n+1}} \\
& \leq C_{n} \varepsilon^{n-p}+\left.\frac{1}{p} v^{p / 2}\right|_{r=\eta_{n+1}}+\left.\delta v^{p / 2}\right|_{r=\eta_{n+1}}+C(\delta) \\
& =C_{n} \varepsilon^{n-p}+\left.\left(\frac{1}{p}+\delta\right) v^{p / 2}\right|_{r=\eta_{n+1}}+C(\delta)
\end{aligned}
$$

from which it follows by choosing $\delta>0$ small enough that

$$
\left.v^{p / 2}\right|_{r=\eta_{n+1}} \leq C_{n} \varepsilon^{n-p}
$$

Noting (3.9), we can see $\sin \rho>0$. Multiply both sides of (3.7) by $\cot \rho=\frac{\cos \rho}{\sin \rho}$ and integrate. Then

$$
-\left.\varepsilon^{p} v^{(p-2) / 2} \rho_{r} \cot \rho\right|_{\eta_{n+1}} ^{1}=\varepsilon^{p} \int_{\eta_{n+1}}^{1} v^{(p-2) / 2} \rho_{r}^{2} \frac{1}{\sin ^{2} \rho} d r+2 \int_{\eta_{n+1}}^{1} \cos ^{2} \rho d r .
$$

Noting $\cot \rho(1)=0$ (which is implied by (3.8)) and $\frac{1}{\sin ^{2} \rho} \geq 1$, we have

$$
\begin{aligned}
& E\left(\rho_{1} ; \eta_{n+1}\right)=\frac{1}{p} \int_{\eta_{n+1}}^{1} v^{p / 2} d r+\frac{1}{\varepsilon^{p}} \int_{\eta_{n+1}}^{1} \cos ^{2} \rho d r \\
\leq & C\left[\int_{\eta_{n+1}}^{1} v^{(p-2) / 2} \rho_{r}^{2} d r+\frac{1}{\varepsilon^{p}} \int_{\eta_{n+1}}^{1} \cos ^{2} \rho d r\right] \leq\left. C v^{(p-2) / 2} \rho_{r} \cot \rho\right|_{r=\eta_{n+1}} .
\end{aligned}
$$


From this, using (3.14)(3.6) and noticing that $n<p$, we obtain

$$
\begin{aligned}
& E\left(\rho_{1} ; \eta_{n+1}\right) \leq\left. C v^{(p-2) / 2} \rho_{r} \cot \rho\right|_{r=\eta_{n+1}} \\
\leq & \left.C v^{(p-1) / 2} \cot \rho\right|_{r=\eta_{n+1}} \leq\left(C_{n} \varepsilon^{n-p}\right)^{(p-1) / p}\left(\frac{C_{n} \varepsilon^{n}}{1-C_{n} \varepsilon^{n}}\right)^{1 / 2} \\
\leq & C_{n+1} \varepsilon^{n+1-p+(n / 2-n / p)} \leq C_{n+1} \varepsilon^{n+1-p} .
\end{aligned}
$$

Define

$$
w_{\varepsilon}=f_{\varepsilon}, \text { for } r \in\left(0, \eta_{n+1}\right) ; \quad w_{\varepsilon}=\rho_{1}, \text { for } r \in\left[\eta_{n+1}, 1\right] \text {. }
$$

Since $f_{\varepsilon}$ is a minimizer of $E_{\varepsilon}(f)$, we have

$$
E_{\varepsilon}\left(f_{\varepsilon}\right) \leq E_{\varepsilon}\left(w_{\varepsilon}\right)
$$

namely,

$$
\begin{aligned}
& E_{\varepsilon}\left(f_{\varepsilon} ; \eta_{n+1}\right) \\
\leq & \frac{1}{p} \int_{\eta_{n+1}}^{1}\left(\rho_{r}^{2}+d^{2} r^{-2} \sin ^{2} \rho\right)^{p / 2} r d r+\frac{1}{\varepsilon^{p}} \int_{\eta_{n+1}}^{1} \cos ^{2} \rho r d r \\
\leq & \frac{C}{p} \int_{\eta_{n+1}}^{1}\left(\rho_{r}^{2}+1\right)^{p / 2} d r+\frac{C}{2 \varepsilon^{p}} \int_{\eta_{n+1}}^{1} \cos ^{2} \rho d r+C \\
= & C E\left(\rho_{1} ; \eta_{n+1}\right)+C .
\end{aligned}
$$

Thus, using (3.15) yields

$$
E_{\varepsilon}\left(f_{\varepsilon} ; \eta_{n+1}\right) \leq C_{n+1} \varepsilon^{n-p+1}
$$

for $\varepsilon \in\left(0, \varepsilon_{0}\right)$. This is just (3.3) for $j=n+1$.

Proposition 3.2. Given $\eta \in(0,1)$. There exist constants $\eta_{N+1} \in\left[\frac{N \eta}{N+1}, \eta\right]$ and $C_{N+1}$ such that

$$
E_{\varepsilon}\left(f_{\varepsilon} ; \eta_{N+1}\right) \leq C_{N+1} \varepsilon^{N-p+1}+\frac{1}{p} \int_{\eta_{N+1}}^{1} \frac{d^{p}}{r^{p-1}} d r
$$

where $N=[p]$.

Proof. Similar to the derivation of (3.6) we may obtain from Proposition 3.1 for $j=N$ that there exists $\eta_{N+1} \in\left[\frac{N \eta}{N+1}, \frac{(N+1) \eta}{N+1}\right]$, such that

$$
\left.\frac{1}{\varepsilon^{p}} \cos ^{2} f_{\varepsilon}\right|_{r=\eta_{N+1}} \leq C_{N} \varepsilon^{N-p}
$$

Also similarly, consider the functional

$$
E\left(\rho, \eta_{N+1}\right)=\frac{1}{p} \int_{\eta_{N+1}}^{1}\left(\rho_{r}^{2}+1\right)^{p / 2} d r+\frac{1}{\varepsilon^{p}} \int_{\eta_{N+1}}^{1} \cos ^{2} \rho d r
$$

whose minimizer $\rho_{2}$ in $W_{f_{\varepsilon}}^{1, p}\left(\left(\eta_{N+1}, 1\right), R^{+}\right)$exists and satisfies

$$
-\varepsilon^{p}\left(v^{(p-2) / 2} \rho_{r}\right)_{r}=\sin 2 \rho, \quad \text { in }\left(\eta_{N+1}, 1\right)
$$




$$
\left.\rho\right|_{r=\eta_{N+1}}=f_{\varepsilon},\left.\quad \rho\right|_{r=1}=f_{\varepsilon}(1)=\frac{\pi}{2}
$$

where $v=\rho_{r}^{2}+1$. From (3.4) for $n=N$ it follows immediately that

$$
\begin{aligned}
E\left(\rho_{2} ; \eta_{N+1}\right) & \leq E\left(f_{\varepsilon} ; \eta_{N+1}\right) \leq C_{N} E_{\varepsilon}\left(f_{\varepsilon} ; \eta_{N+1}\right) \\
& \leq C_{N} E_{\varepsilon}\left(f_{\varepsilon} ; \eta_{N}\right) \leq C_{N} \varepsilon^{N-p} .
\end{aligned}
$$

Similar to the proof of (3.14) and (3.15), we get from (3.17) that

$$
\begin{gathered}
\left.v^{p / 2}\right|_{r=\eta_{N+1}} \leq C_{N} \varepsilon^{N-p}, \\
E\left(\rho_{2} ; \eta_{N+1}\right) \leq C_{N+1} \varepsilon^{N+1-p} .
\end{gathered}
$$

Now we define

$$
w_{\varepsilon}=f_{\varepsilon}, \text { for } r \in\left(0, \eta_{N+1}\right) ; \quad w_{\varepsilon}=\rho_{2}, \text { for } r \in\left[\eta_{N+1}, 1\right]
$$

and then we have

$$
E_{\varepsilon}\left(f_{\varepsilon}\right) \leq E_{\varepsilon}\left(w_{\varepsilon}\right)
$$

Notice that

$$
\begin{aligned}
& \int_{\eta_{N+1}}^{1}\left(\rho_{r}^{2}+d^{2} r^{-2} \sin ^{2} \rho\right)^{p / 2} r d r-\int_{\eta_{N+1}}^{1}\left(d^{2} r^{-2} \sin ^{2} \rho\right)^{p / 2} r d r \\
= & \left.\frac{p}{2} \int_{\eta_{N+1}}^{1} \int_{0}^{1}\left[\left(\rho_{r}^{2}+d^{2} r^{-2} \sin ^{2} \rho\right) s+\left(d^{2} r^{-2} \sin ^{2} \rho\right)(1-s)\right]{ }^{(p-2) / 2}\right] d s \rho_{r}^{2} r d r \\
\leq & C \int_{\eta_{N+1}}^{1} \int_{0}^{1}\left[\left(\rho_{r}^{2}+d^{2} r^{-2} \sin ^{2} \rho\right)^{(p-2) / 2} s^{(p-2) / 2}\right. \\
& \left.+\left(d^{2} r^{-2} \sin ^{2} \rho\right)^{(p-2) / 2}(1-s)^{(p-2) / 2}\right] d s \rho_{r}^{2} r d r \\
= & C \int_{\eta_{N+1}}^{1}\left(\rho_{r}^{2}+d^{2} r^{-2} \sin ^{2} \rho\right)^{(p-2) / 2} \rho_{r}^{2} r d r \int_{0}^{1} s^{(p-2) / 2} d s \\
& +C \int_{\eta_{N+1}^{1}}^{1}\left(d^{2} r^{-2} \sin ^{2} \rho\right)^{(p-2) / 2} \rho_{r}^{2} r d r \int_{0}^{1}(1-s)^{(p-2) / 2} d s \\
\leq & C\left(\int_{\eta_{N+1}}^{1} \rho_{r}^{p} d r+\int_{\eta_{N+1}}^{1} \rho_{r}^{2} d r\right) \leq C \int_{\eta_{N+1}}^{1}\left(\rho_{r}^{2}+1\right)^{p / 2} d r .
\end{aligned}
$$

Hence

$$
\begin{aligned}
& E_{\varepsilon}\left(f_{\varepsilon} ; \eta_{N+1}\right) \leq \frac{1}{p} \int_{\eta_{N+1}}^{1}\left(\left(\rho_{2}\right)_{r}^{2}+d^{2} r^{-2}\left(\sin \rho_{2}\right)^{2}\right)^{p / 2} r d r \\
& +\frac{1}{2 \varepsilon^{p}} \int_{\eta_{N+1}}^{1}\left(\cos \rho_{2}\right)^{2} r d r \\
\leq & \frac{1}{p} \int_{\eta_{N+1}^{1}}^{1}\left(d^{2} r^{-2} \sin ^{2} \rho\right)^{p / 2} r d r+\frac{C}{2 \varepsilon^{p}} \int_{\eta_{N+1}}^{1}\left(\cos \rho_{2}\right)^{2} d r \\
& +C \int_{\eta_{N+1}^{1}}^{1}\left(\left(\rho_{2}\right)_{r}^{2}+1\right)^{p / 2} d r \\
\leq & \frac{1}{p} \int_{\eta_{N+1}^{1}}^{1} r\left(d^{2} r^{-2}\right)^{p / 2} d r+C E\left(\rho_{2} ; \eta_{N+1}\right) .
\end{aligned}
$$


Using (3.19) we have

$$
E_{\varepsilon}\left(f_{\varepsilon} ; \eta_{N+1}\right) \leq \frac{1}{p} \int_{\eta_{N+1}}^{1} r\left(d^{2} r^{-2}\right)^{p / 2} d r+C_{N+1} \varepsilon^{N-p+1} .
$$

This is my conclusion.

4. Proof of Theorem 1.2. Theorem 4.1. Let $u_{\varepsilon}=\left(\sin f_{\varepsilon}(r) e^{i d \theta}, \cos f_{\varepsilon}(r)\right)$ be a minimizer of $E_{\varepsilon}(u, B)$ in $W$. Then

$$
\begin{gathered}
\lim _{\varepsilon \rightarrow 0} f_{\varepsilon}=\frac{\pi}{2}, \quad \text { in } \quad W^{1, p}((\eta, 1], R) \\
\lim _{\varepsilon \rightarrow 0} u_{\varepsilon}=\left(e^{i d \theta}, 0\right),
\end{gathered}
$$

for any $\eta \in(0,1)$ and compact subset $K \subset \bar{B} \backslash\{0\}$.

Proof. It suffices to prove (4.2), since (4.2) implies (4.1). Without loss of generality, we may assume $K=B \backslash B\left(0, \eta_{N+1}\right)$. From Proposition 3.2, We have

$$
E_{\varepsilon}\left(u_{\varepsilon}, K\right)=2 \pi E_{\varepsilon}\left(f_{\varepsilon}, \eta_{N+1}\right) \leq C
$$

where $C$ is independent of $\varepsilon$, namely

$$
\begin{gathered}
\int_{K}\left|\nabla u_{\varepsilon}\right|^{p} d x \leq C, \\
\int_{K}\left|u_{\varepsilon 3}\right|^{2} d x \leq C \varepsilon^{p} .
\end{gathered}
$$

(4.3) and $\left|u_{\varepsilon}\right|=1$ imply the existence of a subsequence $u_{\varepsilon_{k}}$ of $u_{\varepsilon}$ and a function $u_{*} \in W^{1, p}\left(K, R^{3}\right)$, such that

$$
\begin{gathered}
\lim _{\varepsilon_{k} \rightarrow 0} u_{\varepsilon_{k}}=u_{*}, \quad \text { weakly in } W^{1, p}\left(K, R^{3}\right) \\
\lim _{\varepsilon_{k} \rightarrow 0} u_{\varepsilon_{k}}=u_{*}, \quad \text { in } C^{\alpha}\left(K, R^{3}\right), \alpha \in\left(0,1-\frac{2}{p}\right) .
\end{gathered}
$$

(4.4) and (4.5) imply $u_{*}=\left(e^{i d \theta}, 0\right)$. Noticing that any subsequence of $u_{\varepsilon}$ has a convergence subsequence and the limit is always $\left(e^{i d \theta}, 0\right)$, we can assert

$$
\lim _{\varepsilon \rightarrow 0} u_{\varepsilon}=\left(e^{i d \theta}, 0\right), \quad \text { weakly in } W^{1, p}\left(K, R^{3}\right) .
$$

From this and the weakly lower semicontinuity of $\int_{K}|\nabla u|^{p}$, using Proposition 3.2, we have

$$
\begin{aligned}
\int_{K}\left|\nabla e^{i d \theta}\right|^{p} d x & \leq \underline{\lim }_{\varepsilon_{k} \rightarrow 0} \int_{K}\left|\nabla u_{\varepsilon}\right|^{p} d x \leq \overline{\lim }_{\varepsilon_{k} \rightarrow 0} \int_{K}\left|\nabla u_{\varepsilon}\right|^{p} d x \\
& \leq C \lim _{\varepsilon \rightarrow 0} \varepsilon^{N+1-p}+2 \pi \int_{\eta_{N+1}}^{1}\left(d^{2} r^{-2}\right)^{p / 2} r d r
\end{aligned}
$$

and hence

$$
\lim _{\varepsilon \rightarrow 0} \int_{K}\left|\nabla u_{\varepsilon}\right|^{p} d x=\int_{K}\left|\nabla e^{i d \theta}\right|^{p} d x
$$

since $\int_{K}\left|\nabla e^{i d \theta}\right|^{p} d x=2 \pi \int_{\eta_{N+1}}^{1}\left(d^{2} r^{-2}\right)^{p / 2} r d r$. Combining this with (4.6)(4.5) complete the proof of (4.2). 
5. Estimates of convergent rate. Assume $K=\bar{B} \backslash B_{R}$, where $R \in(0,1 / 2)$. We will prove the following

THEOREM 5.1. Let $u_{\varepsilon}(x)=\left(\sin f_{\varepsilon}(r) e^{i d \theta}, \cos f_{\varepsilon}(r)\right)$ be a radial minimizer of $E_{\varepsilon}(u, B)$ on $W$. Then there exist $C, \varepsilon_{0}>0$ such that as $\varepsilon \in(0,1)$,

$$
\begin{gathered}
\int_{R}^{1} r\left[\left(f_{\varepsilon}^{\prime}\right)^{p}+\frac{1}{\varepsilon^{p}} \cos ^{2} f_{\varepsilon}\right] d r \leq C \varepsilon^{p} . \\
\sup _{x \in K}\left|u_{\varepsilon 3}(x)\right| \leq C \varepsilon^{\frac{p-2}{2}} .
\end{gathered}
$$

(5.1) gives the estimate of the rate of $f_{\varepsilon}$ 's convergence to $\pi / 2$ in $W^{1, p}(\eta, 1]$ sense, and that of convergence of $\left|u_{\varepsilon 3}(x)\right|$ to 0 in $C^{0}(K)$ sense is showed by (5.2).

Proof. First, it follows from Jensen's inequality that

$$
\begin{aligned}
2 \pi E_{\varepsilon}\left(f_{\varepsilon} ; \eta\right) & =\frac{2 \pi}{p} \int_{\eta}^{1}\left[\left(f_{\varepsilon}^{\prime}\right)^{2}+\frac{d^{2}}{r^{2}} \sin ^{2} f_{\varepsilon}\right]^{p / 2} r d r+\frac{\pi}{\varepsilon^{p}} \int_{\eta}^{1} \cos ^{2} f_{\varepsilon} r d r \\
& \geq \frac{2 \pi}{p} \int_{\eta}^{1}\left(f_{\varepsilon}^{\prime}\right)^{p} r d r+\frac{\pi}{\varepsilon^{p}} \int_{\eta}^{1} \cos ^{2} f_{\varepsilon} r d r+\frac{2 \pi}{p} \int_{\eta}^{1} \frac{d^{p}}{r^{p}} \sin ^{p} f_{\varepsilon} r d r
\end{aligned}
$$

Combining this with (3.16) yields

$$
\begin{aligned}
& \frac{2 \pi}{p} \int_{\eta}^{1}\left(f_{\varepsilon}^{\prime}\right)^{p} r d r+\frac{\pi}{\varepsilon^{p}} \int_{\eta}^{1} \cos ^{2} f_{\varepsilon} r d r \\
\leq & \frac{2 \pi}{p} \int_{\eta}^{1} \frac{d^{p}}{r^{p}}\left(1-\sin ^{p} f_{\varepsilon}\right) r d r+C \varepsilon^{[p]+1-p} .
\end{aligned}
$$

Noticing that

$$
1-\sin ^{p} f_{\varepsilon} \leq C\left(1-\sin ^{2} f_{\varepsilon}\right)=C \cos ^{2} f_{\varepsilon},
$$

and (4.4), we obtain

$$
\begin{aligned}
& \frac{2 \pi}{p} \int_{\eta}^{1}\left(f_{\varepsilon}^{\prime}\right)^{p} r d r+\frac{\pi}{\varepsilon^{p}} \int_{\eta}^{1} \cos ^{2} f_{\varepsilon} r d r \\
\leq & C \int_{\eta}^{1} \frac{d^{p}}{r^{p}} \cos ^{2} f_{\varepsilon} r d r+C \varepsilon^{[p]+1-p} \\
\leq & C \varepsilon^{p}+C \varepsilon^{[p]+1-p} \leq C \varepsilon^{[p]+1-p} .
\end{aligned}
$$

Using (3.16), (5.3) and the integral mean value theorem we can see that there exists $\eta_{1} \in[\eta, \eta(1+1 / 2)] \subset[R / 2, R]$ such that

$$
\begin{gathered}
{\left[\left(f_{\varepsilon}\right)_{r}^{2}+d^{2} r^{-2} \sin ^{2} f_{\varepsilon}\right]_{r=\eta_{1}} \leq C_{1},} \\
{\left[\frac{1}{\varepsilon^{p}} \cos ^{2} f_{\varepsilon}\right]_{r=\eta_{1}} \leq C_{1} \varepsilon^{[p]-p+1} .}
\end{gathered}
$$

Consider the functional

$$
E\left(\rho, \eta_{1}\right)=\frac{1}{p} \int_{\eta_{1}}^{1}\left(\rho_{r}^{2}+1\right)^{p / 2} d r+\frac{1}{2 \varepsilon^{p}} \int_{\eta_{1}}^{1} \cos ^{2} \rho d r .
$$


It is easy to prove that the minimizer $\rho_{3}$ of $E\left(\rho, \eta_{1}\right)$ in $W_{f_{\varepsilon}}^{1, p}\left(\left(\eta_{1}, 1\right), R^{+}\right)$exists.

By the same way to proof of (3.15), using (5.4) and (5.5) we have

$$
\begin{aligned}
& E\left(\rho_{3}, \eta_{1}\right) \leq\left. v^{\frac{p-2}{2}} \rho_{3 r} \cot \rho_{3}\right|_{r=\eta_{1}} \\
\leq & C_{1} \cot \rho_{3}\left(\eta_{1}\right) \leq C \varepsilon^{\frac{[p]+1-p}{2}+\frac{p}{2}} .
\end{aligned}
$$

Hence, similar to the derivation of (3.16), we obtain

$$
E_{\varepsilon}\left(f_{\varepsilon} ; \eta_{1}\right) \leq C \varepsilon^{\frac{[p]-p+1}{2}+\frac{p}{2}}+\frac{1}{p} \int_{\eta_{1}}^{1} \frac{d^{p}}{r^{p-1}} d r
$$

Thus (5.3) may be rewritten as

$$
\begin{gathered}
\int_{\eta_{1}}^{1}\left(f_{\varepsilon}^{\prime}\right)^{p} r d r+\frac{1}{\varepsilon^{p}} \int_{\eta_{1}}^{1} \cos ^{2} f_{\varepsilon} r d r \\
\leq C \varepsilon^{\frac{[p]+1-p}{2}+\frac{p}{2}}+C \varepsilon^{p} \leq C_{2} \varepsilon^{\frac{[p]+1-p}{2}+\frac{p}{2}} .
\end{gathered}
$$

Let $\eta_{m}=R\left(1-\frac{1}{2^{m}}\right)$ where $R<1$. Proceeding in the way above (whose idea is improving the exponent of $\varepsilon$ from $\frac{[p]+1-p}{2^{k}}+\frac{\left(2^{k}-1\right) p}{2^{k}}$ to $\frac{[p]+1-p}{2^{k+1}}+\frac{\left(2^{k+1}-1\right) p}{2^{k+1}}$ step by step), we can get that for any $m \in N$,

$$
\int_{\eta_{m}}^{1}\left(f_{\varepsilon}^{\prime}\right)^{p} r d r+\frac{1}{\varepsilon^{p}} \int_{\eta_{m}}^{1} \cos ^{2} f_{\varepsilon} r d r \leq C \varepsilon^{\frac{[p]+1-p}{2^{m}}+\frac{\left(2^{m}-1\right) p}{2^{m}}}+C \varepsilon^{p} .
$$

Letting $m \rightarrow \infty$, we derive

$$
\int_{R}^{1}\left(f_{\varepsilon}^{\prime}\right)^{p} r d r+\frac{1}{\varepsilon^{p}} \int_{R}^{1} \cos ^{2} f_{\varepsilon} r d r \leq C \varepsilon^{p} .
$$

This is (5.1).

From (5.1) we can see that

$$
\int_{K} u_{\varepsilon 3}^{2} d x \leq C \varepsilon^{2 p} .
$$

On the other hand, for any $x_{0} \in K$, we have

$$
\left|u_{\varepsilon 3}(x)-u_{\varepsilon 3}\left(x_{0}\right)\right| \leq C \varepsilon^{(2-p) / p}\left|x-x_{0}\right|^{1-2 / p}, \quad \forall x \in B\left(x_{0}, \alpha \varepsilon\right),
$$

by applying Proposition 2.2, where $\alpha=\left(\frac{\left|u_{\varepsilon 3}\left(x_{0}\right)\right|}{2 C}\right)^{\frac{p}{p-2}}$. Thus

$$
\left|u_{\varepsilon 3}(x)\right| \geq\left|u_{\varepsilon 3}\left(x_{0}\right)\right|-C \alpha^{1-2 / p} \geq \frac{1}{2}\left|u_{\varepsilon 3}\left(x_{0}\right)\right| .
$$

Substituting this into (5.6) we obtain

$$
\begin{aligned}
& C \varepsilon^{2 p} \geq \int_{K} u_{\varepsilon 3}^{2} d x \geq \int_{B\left(x_{0}, \alpha \varepsilon\right)} u_{\varepsilon 3}^{2} d x \\
& \geq \frac{\pi}{4}\left|u_{\varepsilon 3}\left(x_{0}\right)\right|^{2}(\alpha \varepsilon)^{2}=\frac{\pi}{4}\left(\frac{1}{2 C}\right)^{\frac{2 p}{p-2}}\left|u_{\varepsilon 3}\left(x_{0}\right)\right|^{2+\frac{2 p}{p-2}} \varepsilon^{2},
\end{aligned}
$$


which implies

$$
\left|u_{\varepsilon 3}\left(x_{0}\right)\right| \leq C \varepsilon^{\frac{p-2}{2}} .
$$

Noting $x_{0}$ is an arbitrary point in $K$, we have

$$
\sup _{x \in K}\left|u_{\varepsilon 3}(x)\right| \leq C \varepsilon^{\frac{p-2}{2}} .
$$

Thus (5.2) is derived and the proof of Theorem is complete.

\section{REFERENCES}

[1] F.Bethuel, H.Brezis, F.Helein, Ginzburg-Landau Vortices, Birkhauser, 1994.

[2] F.B.HAnG, F.H.Lin, Static theory for planar ferromagnets and antiferromagnets, Acta. Math. Sinica, English Series, 17(2001), pp. 541-580.

[3] S.Komineas, N.Papanicolaou, Vortex dynamics in two-dimensional antiferromagnets, Nonlinearity, 11(1998), pp. 265-290.

[4] N.Papanicolaou, P.N.Spathis, Semitopological solutions in planar ferromagnets, Nonlinearity, 12(1999), pp. 285-302. 